\title{
Oscillations of Respiration and Adenine Nucleotides in Synchronous Cultures of Acanthamoeba castellanii: Mitochondrial Respiratory Control in vivo
}

\author{
By STEVEN W. EDWARDS AND DAVID LLOYD \\ Department of Microbiology, University College, \\ Newport Road, Cardiff CF2 1TA
}

(Received 17 May 1978)

\begin{abstract}
Synchronous cultures of the soil amoeba Acanthamoeba castellanii, prepared by a size selection procedure involving minimum metabolic perturbation, divided with a high degree of synchrony and showed a discrete S-phase of DNA synthesis. Oxygen uptake rates doubled overall during one cell cycle time of 7 to $8 \mathrm{~h}$ but rose to seven distinct maxima during this period. Control experiments showed that the selection procedure did not give rise to the oscillations. The maxima of respiration were more sensitive to inhibition by cyanide than were the minima of respiration; the effect of carbonyl cyanide $p$-trifluoromethoxyphenylhydrazone (an uncoupler of oxidative phosphorylation) was, however, greater at respiratory minima, than at the maxima. Pool levels of ATP, ADP and AMP also oscillated during the cell cycle with maximum amplitudes (peak-trough, \% minimal values) of 108, 194 and 520, respectively. Adenylate charge values varied between 0.63 and 0.88. Respiratory maxima were in phase with maximum ADP levels but out of phase with maxima of ATP/ADP ratios. These results suggest that the overall changes in respiration rates during the cell cycle of $A$. castellanii are the result of in vivo respiratory control.
\end{abstract}

\section{INTRODUCTION}

The rate of respiration of isolated functionally intact mitochondria depends on the level of ADP (Chance \& Williams, 1956). These authors defined the steady states of mitochondrial respiration: State 4 is an aerobic state characterized by a low respiration rate (a 'resting' state) even though respiratory substrate is available, and State 3 is the 'active' state of rapid respiration and phosphorylation, with adequate supplies of substrate and phosphate acceptor. Transitions from State 3 to State 4 have been demonstrated in starved yeast cells when the endogenous pool of ADP becomes exhausted during respiration of added ethanol (Chance, $1959 a, b$ ), and State 4 to State 3 transitions may be demonstrated in this system by way of hexokinase-produced ADP on addition of glucose. In ascites tumour cells, it is evident that ADP rather than inorganic phosphate controls the respiration rate (Hess \& Chance, 1959). Addition of uncouplers to mitochondria in the resting state abolishes the link between the respiratory electron transport chain and the associated reactions of energy conservation, and leads to a state of rapid respiration [(State $3 \mathrm{u})$, Chance \& Hollunger, 1963; Chance et al., 1963].

Although mitochondrial respiratory control has previously been demonstrated in vivo, transitions between States 3 and 4 have never been shown to occur as a necessary feature of energy metabolism driving the biosynthetic reactions of cell growth. In this report we show that the respiratory oscillations which occur during the cell cycle in synchronous cultures of the soil amoeba Acanthamoeba castellanii represent in vivo mitochondrial respiratory 
control. Thus the organism oscillated between States 3 and 4 with a mean frequency of $1.0 \mathrm{~h}^{-1}$ and peak to trough amplitude of ADP between 43 and $194 \%$ of the minimum values during a mean cell cycle time of $7 \cdot 8 \mathrm{~h}$.

\section{METHODS}

Organism and growth conditions. Acanthamoeba castellanii was maintained and grown with shaking at $30^{\circ} \mathrm{C}$ as described previously (Griffiths \& Chagla, 1972; Edwards et al., 1977). Cells were counted in a Fuchs-Rosenthal haemocytometer slide (Baird \& Tatlock, Chadwell Heath, Romford, Essex) after suitable dilution in $50 \mathrm{~mm}-\mathrm{MgCl}_{2}$, pH 7.4.

Preparation of synchronous cultures. Synchronous cultures were produced by centrifuging exponentially growing cultures in sterile, stoppered centrifuge tubes at $300 \mathrm{rev} . \min ^{-1}\left(10 \mathrm{~g}, r_{\mathrm{av}} .10 .0 \mathrm{~cm}\right)$ for $2 \mathrm{~min}$ in the $4 \times 50 \mathrm{ml}$ swing-out head of an MSE bench centrifuge (Chagla \& Griffiths, 1978). After centrifugation the supernatant, which consisted of the slowest sedimenting cells suspended in growth medium (approx. $10 \%$ of the original exponentially growing culture), was carefully decanted into a conical flask and grown as a synchronous culture. The degree of synchrony was assessed by the synchrony index $(F)$ of Blumenthal \& Zahler (1962), which has a value of 1.0 for a theoretically perfect synchronous culture and 0 for an exponentially growing culture.

Analytical methods. DNA was estimated by the fluorimetric method of Kissane \& Robins (1958) with calf thymus DNA as standard. Oxygen uptake rates were measured polarographically at $30^{\circ} \mathrm{C}$ in a Clark oxygen electrode (Lloyd \& Brookman, 1967) using undiluted samples of cells suspended in growth medium. Cyanide was added as an aqueous solution ( $\mathrm{pH} 7 \cdot 4)$ and carbonyl cyanide $p$-trifluoromethoxyphenylhydrazone (FCCP) was added as an ethanolic solution (the concentration of solvent alone had no effect on respiration). Adenine nucleotide pool levels were measured by the luciferase method (Chapman et al., 1971), as described previously (Edwards et al., 1975), after rapid quenching and extraction in boiling chloroform. Adenylate charge (Atkinson \& Walton, 1967) was calculated as:

$$
\text { Adenylate charge }=\frac{\mathrm{ATP}+\frac{1}{2} \mathrm{ADP}}{\mathrm{ATP}+\mathrm{ADP}+\mathrm{AMP}}
$$

Chemicals. Calf thymus DNA (type V, sodium salt), buffered firefly lantern extracts, myokinase (grade III, rabbit muscle), pyruvate kinase (type II, rabbit skeletal muscle), phosphoenolpyruvic acid (sodium salt) and ATP (disodium salt) were from Sigma. FCCP was from Boehringer. All other chemicals were of analytical grade.

\section{RESULTS AND DISCUSSION}

Size-selection of $A$. castellanii by low-speed centrifugation of exponentially growing cultures provides a gentle and rapid method for the production of synchronous cultures which show a complete doubling in cell numbers. There was some variation in the lag before entry into cell division in different experiments, as it was difficult to control centrifugation conditions precisely enough to ensure selection of an identical proportion of the total population on each occasion. However, the cultures exhibited a high degree of synchrony ( $F=0.73 \pm 0.05,14$ experiments) and the cell cycle time, taken as the period from time zero to the time when all the cells had divided $(7.83 \pm 0.54 \mathrm{~h}, 14$ experiments), was slightly shorter than the generation time of exponentially growing cultures $(8 \cdot 52 \pm 0 \cdot 82 \mathrm{~h}$, 12 experiments).

The changes in total DNA during synchronous growth are shown in Fig. 1. The DNA concentration remained constant at about 2.5 pg per cell until $3.75 \mathrm{~h}$ and then doubled by $5 \cdot 2 \mathrm{~h}$.

Figure $2(b)$ shows the time course of the changes in respiration rate through the first cell cycle after selection of a population of small cells from an exponentially growing culture of A. castellanii. Cell numbers showed a perfect doubling, and cell division was completed after $7 \mathrm{~h}$, with a synchrony index of 0.64 . The mean respiration rate of the synchronous culture doubled over this period and showed seven maxima, the peak to trough amplitude of the oscillation accounting for 18 to $31 \%$ of the minimum respiration rate. Addition of $0.5 \mathrm{~mm}-\mathrm{KCN}$ to samples withdrawn from the culture gave differing effects at different times during the cell cycle; Fig. $2(b, c)$ shows that the greatest inhibitory effect occurred when 

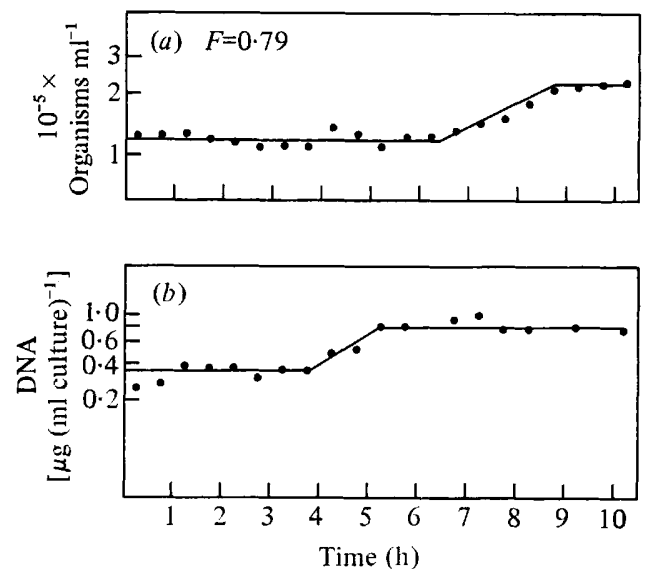

Fig. 1. Changes in DNA content of a synchronous culture of $A$. castellanii. The synchronous culture contained $5 \%$ of the original population of the exponentially growing culture.

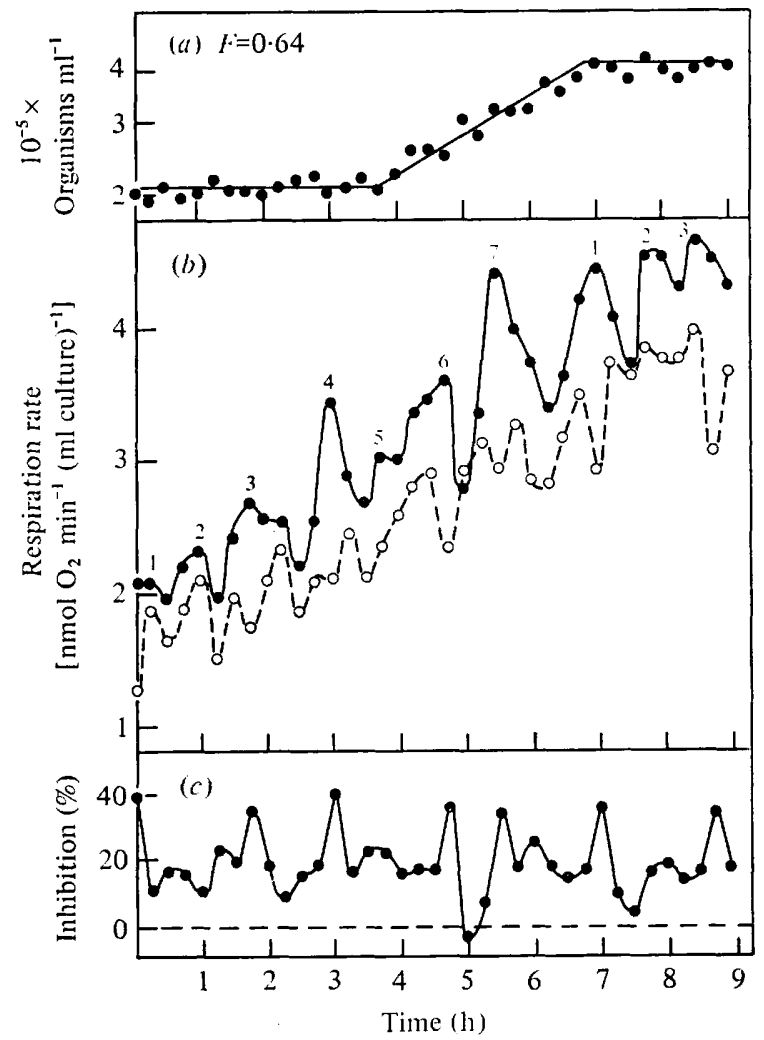

Fig. 2. Effect of cyanide on respiratory oscillations in a synchronously dividing culture of $A$. castellanii. The synchronous culture contained $15 \%$ of the exponentially growing culture. At $15 \mathrm{~min}$ intervals, samples were withdrawn from the culture and rates of oxygen uptake were measured in the presence or absence of $0.5 \mathrm{~mm}$-cyanide. (a) Cell numbers and synchrony index, $F$; (b) respiration rate in the presence $(O)$ or absence $(\bullet)$ of 0.5 mM-cyanide; $(c)$ inhibition of respiration (\%) resulting from the addition of cyanide. 


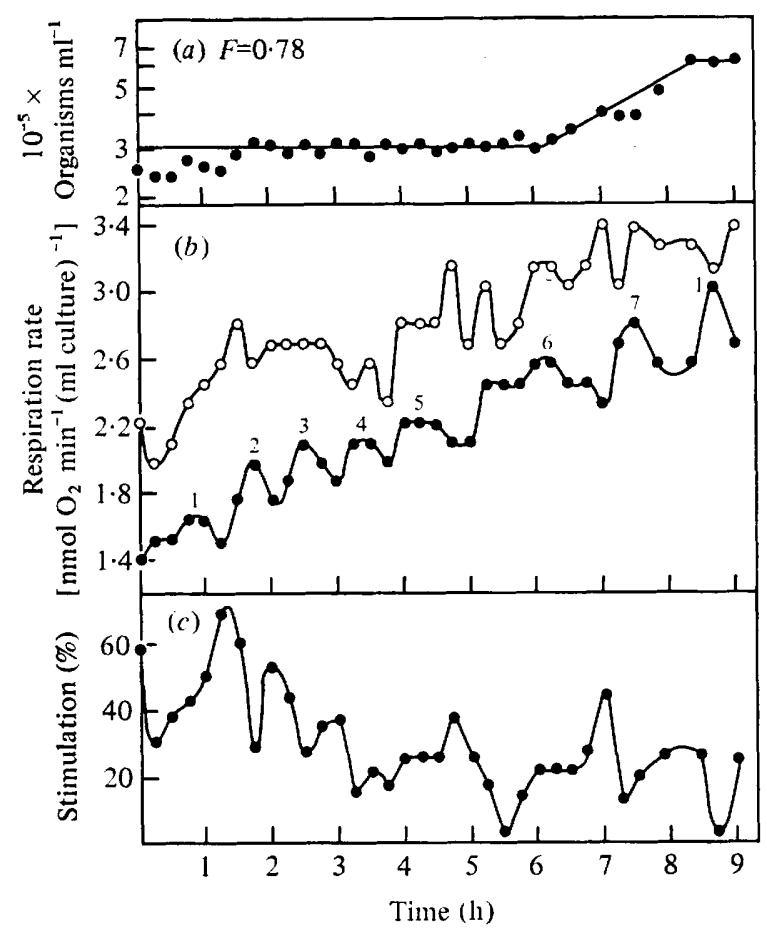

Fig. 3. Effect of FCCP on respiratory oscillations in a synchronously dividing culture of A. castellanii. The synchronous culture contained $9 \%$ of the exponentially growing culture. (a) Cell numbers and synchrony index, $F ;(b)$ respiration rate in the presence $(O)$ or absence (๑) of $2.36 \mu \mathrm{M}-\mathrm{FCCP} ;(c)$ stimulation of respiration (\%) resulting from the addition of FCCP.

cyanide was added to organisms showing maximum respiratory activities and was least at troughs of respiration. Mitochondria isolated from exponentially growing cell populations showed respiratory control with 2-oxoglutarate, succinate and NADH as respiratory substrates, and in all cases $1 \mathrm{~mm}$-cyanide produced a greater degree of inhibition with mitochondria in State 3 than in State 4 [e.g. for succinate oxidation, State 3 inhibition $100 \%$, State 4 inhibition 57 to $82 \%$ (Edwards \& Lloyd, 1978)]. Addition of $2.36 \mu \mathrm{M}-\mathrm{FCCP}$ (an uncoupler of oxidative phosphorylation) to samples withdrawn from a synchronous culture at different stages of the cell cycle gave a preferential stimulatory effect on respiration rates when organisms showed minimum respiratory activity (Fig. $3 b, c$ ).

That the respiratory oscillations are not a consequence of metabolic perturbation produced by the centrifugal selection procedure is shown in Fig. 4. An exponentially growing culture was centrifuged under the same conditions as used to produce synchronous cultures, but after centrifuging the contents of the tube were remixed and the entire cell population was grown as an asynchronous culture (Fig. $4 a$ ). This culture, which received the same treatment as the synchronous cultures, showed a smooth increase in respiration rates even in the earliest samples taken; there was no indication of oscillatory behaviour (Fig. $4 b$ ).

Pool sizes of adenine nucleotides also oscillated in a synchronous culture and the periodicity of the oscillations was similar to that observed for respiration rate (Fig. 5). The maximum observed amplitudes (peak-trough) for ATP, ADP and AMP, expressed as a percentage of the minimum values, were 108, 194 and 520, respectively. Adenylate charge values varied between 0.63 and 0.88 and the ratio ATP/ADP varied between values of 0.9 and 10.9. The phase relationships between respiratory maxima, ADP and ATP/ADP maxima are shown on a cell cycle map in which a single traverse of the cycle is represented as a circle following cell division at $0^{\circ}$ (Fig. 6). Thus maxima of respiratory activity 


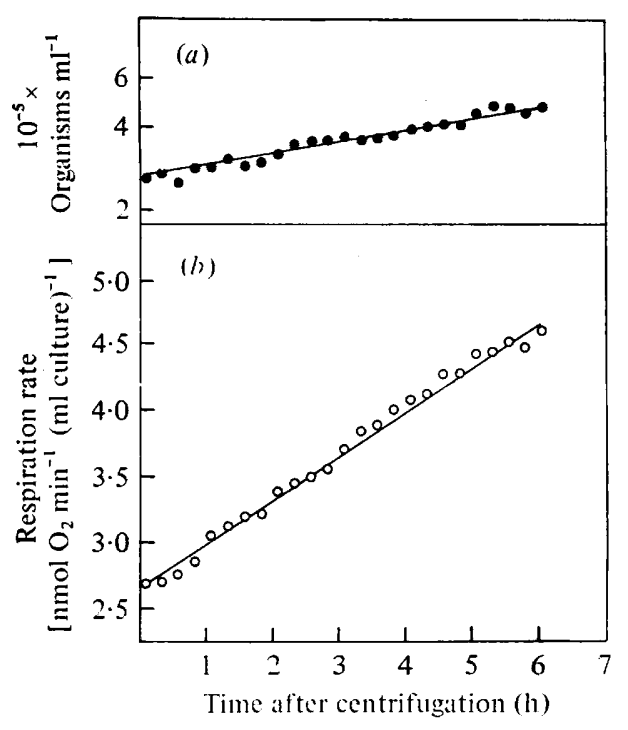

Fig. 4

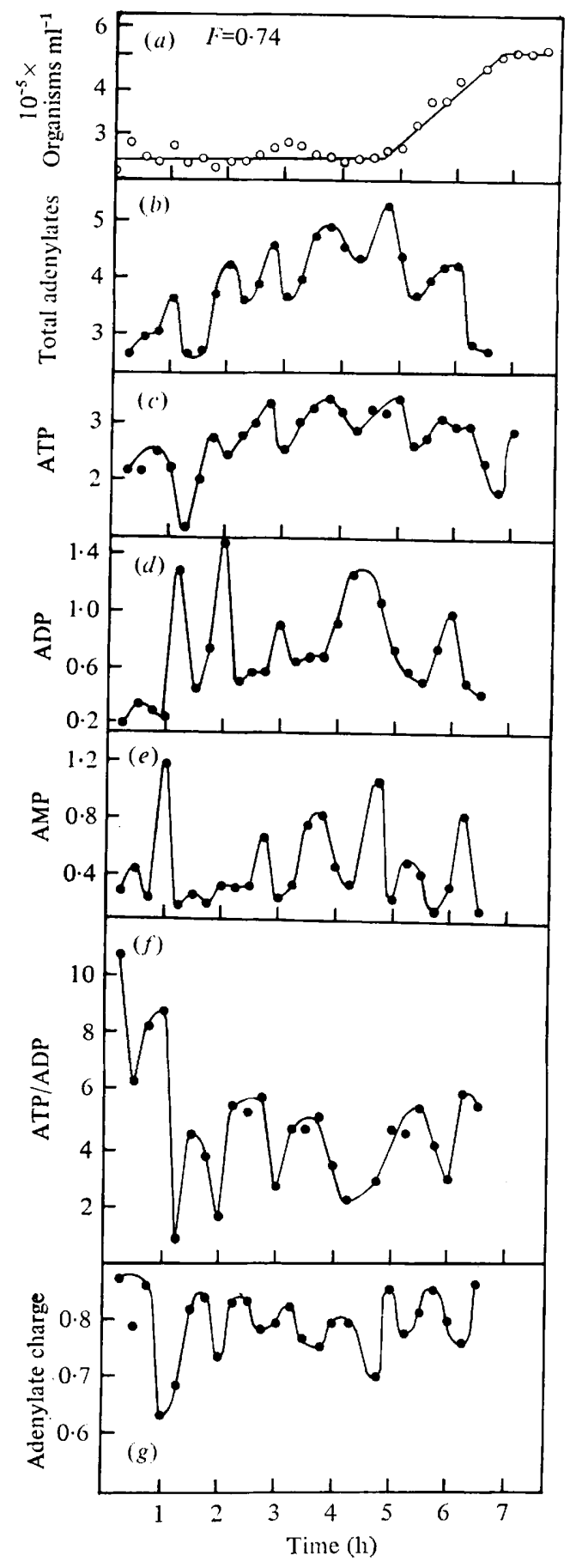

Fig. 5

Fig. 4. Changes in cell numbers $(a)$ and oxygen uptake rates $(b)$ after centrifugation and resuspension of an exponentially growing culture. The exponentially growing culture was centrifuged at $300 \mathrm{rev}$. $\min ^{-1}\left(10 \mathrm{~g}, r_{\mathrm{ar}}, 10 \mathrm{~cm}\right)$ for $2 \mathrm{~min}$, and then remixed and returned to the growth vessel.

Fig. 5. Changes in adenine nucleotide pool levels and adenylate charge values in a synchronously dividing culture of $A$. castellanii. The synchronous culture contained $10 \%$ of the exponentially growing population; $1 \mathrm{ml}$ samples were withdrawn at $15 \mathrm{~min}$ intervals for measurements of adenine nucleotides after rapid quenching and extraction in chloroform. (a) Cell numbers and synchrony index, $F$; $(b)$ total adenylates; $(c)$ ATP; $(d)$ ADP; $(e)$ AMP; $(f)$ ATP/ADP ratio; $(g)$ adenylate charge. Adenylate concentrations are expressed as nmol (ml culture) ${ }^{-1}$. 


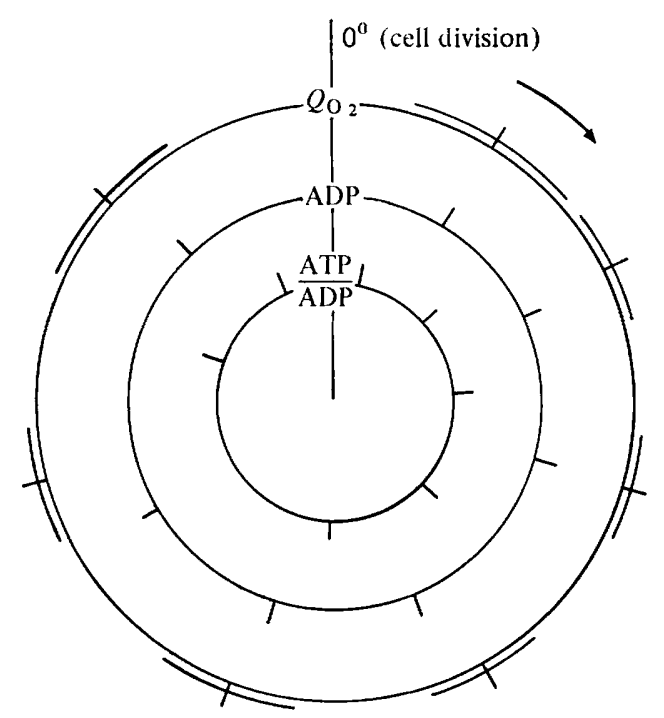

Fig. 6. Timings of cell cycle events in A. castellanii. The cell cycle is represented as a cycle from $0^{\circ}$ to $360^{\circ}: 0^{\circ}$ represents time zero in a synchronous culture, and $360^{\circ}$ the time when all the cells have completed division. Maxima of cell cycle events are shown as phase angles with respect to their timings in synchronous cultures. Timings of respiratory maxima are mean values of five experiments, with standard deviations.

correspond to maximum ADP levels and are out of phase with maxima of ATP/ADP ratios. Therefore at those times in the cell cycle when respiration is at a maximum, growing organisms are in State 3, whereas organisms showing minimum respiratory activity are in State 4. Transitions of respiratory activity observed during the cell cycle of the bacterium Alcaligenes eutrophus have also been explained in terms of differing energy requirement at different stages of the cell cycle (Edwards \& Jones, 1977).

These respiratory oscillations are clearly different from those previously observed in synchronous cultures of the fission yeast Schizosaccharomyces pombe (growing in the presence of glucose), where a Pasteur effect was invoked to explain the modulation of mitochondrial activity (Poole et al., 1973), in the trypanosomatid protozoon Crithidia fasciculata (Edwards et al., 1975), or in Tetrahymena pyriformis (Lloyd et al., 1978). The observation of a dramatic fluctuation of adenylate energy charge in the trypanosome (Edwards et al., 1975), in T. pyriformis (Lloyd et al., 1978), and in A. castellanii (this report) is of great interest as it has been suggested that thermodynamic and kinetic considerations of the regulatory mechanisms involved in energy metabolism would predict considerable constraints on variations of this parameter, and a tendency to stabilization of adenylate charge between values of 0.87 and 0.95 (Chapman \& Atkinson, 1977). That variations in adenylates on a time scale comparable with those of epigenetic oscillations (Goodwin, 1976) have not previously been reported is due to the fact that time-averaging occurs in all experiments with populations of organisms growing asynchronously.

Although we have partially time-resolved the fluctuations in adenylates, it must be pointed out that the values obtained still represent a spatial average, and take no account of intracellular compartmentation.

The periodicities of the oscillations during the cell cycle of $A$. castellanii are between one and two orders of magnitude longer than typical 'metabolic' oscillations (Pye \& Chance, 1966). Presumably suitably frequent sampling might also reveal a high-frequency oscillation superimposed on the waveforms presented. The method employed in the present work for producing synchronous cultures is 'minimally perturbing' in that the organisms 
do not leave their growth medium and therefore are not subject to transient nutrient or oxygen deprivation. They are merely exposed to a relative centrifugal force of $10 \mathrm{~g}$ for $2 \mathrm{~min}$. The cultures show a discrete S-phase of DNA synthesis, and therefore they are synchronous with respect to both growth and division, and DNA replication cycles. That the respiratory oscillations are not observed in control experiments with identically treated asynchronous cultures, and that they show no signs of attenuation over $9 \mathrm{~h}$ of synchronous growth, shows that they are genuinely associated with the sequence of cell cycle events.

Further work is necessary to determine: (i) the relationship of the observed oscillations of energy metabolism to changes in rates of biosynthetic reactions and/or activities of ATPases which presumably accompany alterations in steady-state pool sizes of ADP; (ii) the significance of the characteristic periodicity of energy metabolism (i.e. seven maxima per cell cycle); (iii) the low frequency [circadian subharmonic? (Delmer \& Brody, 1975)] waveform observed as a 'carrier-wave' on the plot of total adenylates (Fig. 5b); (iv) intraand extra-mitochondrial compartmentation of adenine nucleotides.

\section{S.W.E. holds a Medical Research Council Postgraduate Studentship.}

\section{REFERENCES}

AtKinson, D. E. \& Walton, G. M. (1967). Adenosine triphosphate conservation in metabolic regulation. Journal of Biological Chemistry 242, 3239-3241.

Blumenthal, L. K. \& Zahler, S. A. (1962). Index for measurement of synchronization of cell populations. Science 135, 724.

Chagla, A. H. \& Griffiths, A. J. (1978). Synchronous cultures of Acanthamoeba castellanii and their use in the study of encystation. Journal of General Microbiology 108, 39-43.

Chance, B. $(1959 a)$. Phosphorylation efficiency of the intact cell. II. Crossover phenomena in Bakers' Yeast. Journal of Biological Chemistry 234, 3036-3040.

Chance, B. $(1959 b)$ ). Phosphorylation efficiency of the intact cell. III. Phosphorylation and dephosphorylation in yeast cells. Journal of Biological Chemistry 234, 3041-3043.

Chance, B. \& Hollunger, G. (1963). Inhibition of electron and energy transfer in mitochondria. $I$. Effects of amytal, thiopental, progesterone and methylene glycol. Journal of Biological Chemistry 278, 418-431.

Chance, B. \& Williams, G. R. (1956). The respiratory chain and oxidative phosphorylation. Advances in Enzymology 17, 65-134.

Chance, B., Williams, G. R. \& Hollunger, G. (1963). Inhibition of electron and energy transfer in mitochondria. III. Spectroscopic and respiratory effects of uncoupling agents. Journal of Biological Chemistry 278, 439-444.

Chapman, A. G. \& Atkinson, D. E. (1977). Adenine nucleotide concentrations and turnover rates. Their correlation with biological activity in bacteria and yeast. Advances in Microbial Physio$\log y 15,253-306$.

Chapman, A. G., Fall, L. \& Atkinson, D. E. (1971). Adenosine triphosphate and synchronous mitosis in Physarum polycephalum. Journal of Bacteriology 96, 330-337.

Delmer, D. P. T. \& Brody, S. (1975). Circadian rhythms in Neurospora crassa. Oscillation in the level of an adenine nucleotide. Journal of Bacterio$\log y$ 121, 548-553.

EDWARDS, C. \& Jones, C. W. (1977). Respiratory properties of synchronous cultures of Alcaligenes eutrophus H16 prepared by a continuous-flow size selection method. Journal of General Microbiology 99, 383-388.

Edward, S. W. \& Lloyd, D. (1978). Properties of mitochondria isolated from cyanide-stimulated and cyanide-sensitive cultures of Acanthamoeba castellanii. Biochemical Journal 174, 203-211.

Edwards, C., Statham, M. \& Lloyd, D. (1975). The preparation of large-scale synchronous cultures of the trypanosomatid, Crithidia fasciculata, by cell-size selection: changes in respiration and adenylate charge through the cell-cycle. Journal of General Microbiology 88, 141-152.

Edwards, S. W., Chagla, A. H., Griffiths, A. J. \& LLOYD, D. (1977). The cytochromes of Acanthamoeba castellanii. Biochemical Journal 168, 113-121.

Goodwin, B. C. (1976). Analytical Physiology of Cells and Developing Organisms. London and New York. Academic Press.

Griffiths, A. J. \& Chagla, A. H. (1972). The growth and encystment of Acanthamoeba castellanii. Journal of Protozoology S19, Abstr. 130.

Hess, B. \& Chance, B. (1959). Phosphorylation efficiency of the intact cell. I. Glucose-oxygen titrations in ascites tumour cells. Journal of Biological Chemistry 234, 3031-3035.

Kissane, J. M. \& Robins, E. (1958). The fluorometric measurement of DNA in animal tissues with special reference to the central nervous system. Journal of Biological Chemistry 233, 184-188.

Lloyd, D. \& Brookman, J. S. G. (1967). An oxygen electrode reaction vessel. Biotechnology and Bioengineering 9, 271-272.

Lloyd, D., Phillips, C. A. \& Statham, M. (1978). Oscillations of respiration, adenine nucleotide 
levels and heat evolution in synchronous cultures of Tetrahymena pyriformis sT prepared by continuous-flow selection. Journal of General Microbiology 106, 19-26.

Poole, R. K., Lloyd, D. \& KemP, R. B. (1973). Respiratory oscillations and heat evolution in synchronously dividing cultures of the fission yeast Schizosaccharomyces pombe $972 \mathrm{~h}^{-}$. Journal of General Microbiology 77, 209-220.

Pye, K. \& Chance, B. (1966). Sustained oscillations of reduced pyridine nucleotide in a cell-free extract of Saccharomyces carlsbergensis. Proceedings of the National Academy of Sciences of the United States of America 55, 888-894. 